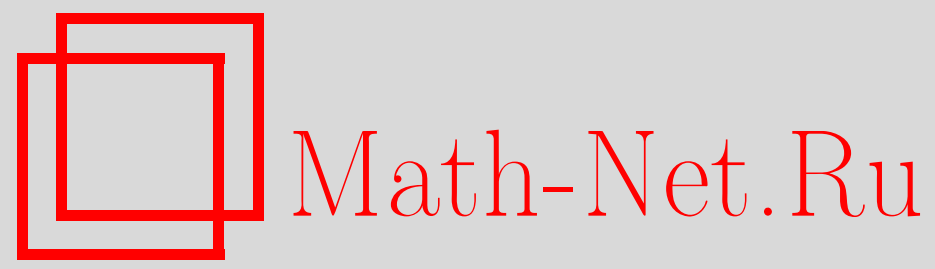

Д. В. Христофоров, Рекуррентные соотношения для аппроксимаций Эрмита-Паде некоторой системы из четырех функций марковского и стилтьесовского типа, Совр. пробл. матем., 2007, выпуск $9,11-26$

DOI: https://doi.org/10.4213/spm13

Использование Общероссийского математического портала MathNet.Ru подразумевает, что вы прочитали и согласны с пользовательским соглашением

http://www . mathnet.ru/rus/agreement

Параметры загрузки:

IP: 3.91 .87 .62

26 апреля 2023 г., 17:07:12

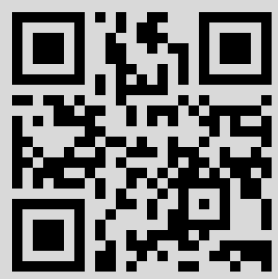




\section{Рекуррентные соотношения для аппроксимаций Эрмита-Паде некоторой системы из четырех функций марковского и стилтьесовского типа ${ }^{1}$} Д. В. Христофоров

\section{1. Введение}

Пусть

$$
w_{k}(x)=x^{\alpha_{k}}(1-x)^{\alpha} e^{\beta x}, \quad k=1,2, \quad \beta<0, \quad \alpha_{1}-\alpha_{2} \notin \mathbb{Z},
$$

- две весовые функции Якоби-Лаггера на $[0, \infty]$, которые определяют систему из четырех функций $\left\{f_{j}\right\}_{j=1}^{4}$ :

$$
f_{1}=\hat{\mu}_{1}, \quad f_{2}=\hat{\mu}_{2}, \quad f_{3}=\widehat{S}_{1}, \quad f_{4}=\widehat{S}_{2},
$$

две из которых функции марковского типа:

$$
\hat{\mu}_{k}(x):=\int_{0}^{1} \frac{w_{k}(x)}{z-x} d x, \quad k=1,2,
$$

две другие функции стилтьесовского типа:

$$
\widehat{S}_{k}(x):=\int_{1}^{\infty} \frac{w_{k}(x)}{z-x} d x, \quad k=1,2
$$

Рациональные аппроксимации Эрмита-Паде $\pi_{\vec{n}}$ для системы $\left\{f_{j}\right\}_{j=1}^{4}$ и векторного индекса $\vec{n}:=\left(n_{1}, n_{2}, n_{3}, n_{4}\right) \in \mathbb{N}^{4}$ определя-

\footnotetext{
${ }^{1}$ Работа частично поддержана грантом научных школ НШ-1551.2003.1 и исследовательским грантом РФФИ-05-01-00522.
}

(c) Христофоров Д.В., 2007 
ются следующим образом (см. [1]):

$$
\begin{aligned}
\pi_{\vec{n}}:=\left\{\frac{P_{\vec{n}}^{(j)}}{Q_{\vec{n}}}\right\}_{j=1}^{4}: \\
\qquad \begin{array}{l}
\operatorname{deg} Q_{\vec{n}} \leqslant|\vec{n}|:=\sum_{j=1}^{4} n_{j}, \\
\left(f_{j} Q_{\vec{n}}-P_{\vec{n}}^{(j)}\right)(z)=: R_{\vec{n}}^{(j)}(z)=O\left(\frac{1}{z^{n_{j}+1}}\right), \quad z \rightarrow \infty .
\end{array}
\end{aligned}
$$

Известно, что общие знаменатели $Q_{\vec{n}}$ рациональных аппроксимаций (3) удовлетворяют системе из четырех типов соотношений ортогональности:

$$
\begin{array}{ll}
\int_{0}^{1} Q_{\vec{n}}(x) x^{\nu} w_{j}(x) d x=0, & \nu=0, \ldots, n_{j}-1, \quad j=1,2, \\
\int_{1}^{\infty} Q_{\vec{n}}(x) x^{\nu} w_{j}(x) d x=0, & \nu=0, \ldots, n_{j}-1, \quad j=1,2 .
\end{array}
$$

Такие многочлены называются совместно ("multiple") ортогональными.

Совместно ортогональные многочлены (4) относительно весов (1), среди других примеров, были рассмотрены в работе [2]. В частности, в [2] приведена формула Родрига для этих многочленов при $\vec{n}=\left(n_{1}, n_{1}, n_{2}, n_{2}\right)=:\left[n_{1}, n_{2}\right]$ :

$$
\begin{aligned}
Q_{\left[n_{1}, n_{2}\right]}(z)= & w_{2}^{-1}(z) \frac{d^{n_{2}}}{d z^{n_{2}}}\left[w_{2}(z) z^{n_{2}} w_{1}^{-1}(z)\right. \\
& \left.\times \frac{d^{n_{1}}}{d z^{n_{1}}}\left[w_{1}(z) z^{n_{1}}(1-z)^{n_{1}+n_{2}}\right]\right] .
\end{aligned}
$$

Известно (см. [3], [4]), что знаменатели аппроксимаций Эрмита-Паде для системы из $p$ функций удовлетворяют $(p+2)$ членному рекуррентному соотношению. При $p=4$ рекуррентное соотношение связывает любые шесть соседних многочленов $Q_{\left(n_{1}, n_{2}, n_{3}, n_{4}\right)}$ в четырехмерной таблице вектор-индексов $\vec{n}$. Нас будут интересовать рекуррентные соотношения, связывающие между собой диагональные и наддиагональные элементы этой таблицы, т.н. "step-line":

$$
\ldots, Q_{\vec{n}-\vec{e}_{4}}, Q_{\vec{n}-\vec{e}_{4}+\vec{e}_{1}}, Q_{\vec{n}-\vec{e}_{4}+\vec{e}_{2}}, Q_{\vec{n}-\vec{e}_{4}+\vec{e}_{3}}, Q_{\vec{n}}, Q_{\vec{n}+\vec{e}_{1}}, \ldots,
$$


здесь мы используем обозначения

$$
\begin{array}{rlrl}
\vec{n}=(n, n, n, n), & \vec{e}_{1}=(1,0,0,0), & \vec{e}_{2}=(1,1,0,0), \\
\vec{e}_{3}=(1,1,1,0), & \vec{e}_{4}=(1,1,1,1) .
\end{array}
$$

Таким образом, для последовательного вычисления многочленов (6) нам требуется набор из четырех шестичленных рекуррентных соотношений, связывающих вектор-индексы:

$$
\begin{array}{llllll}
\vec{n}-\vec{e}_{4} & \vec{n}-\vec{e}_{4}+\vec{e}_{1} & \vec{n}-\vec{e}_{4}+\vec{e}_{2} & \vec{n}-\vec{e}_{4}+\vec{e}_{3} & \vec{n} & \vec{n}+\vec{e}_{1} \\
\vec{n}-\vec{e}_{4}+\vec{e}_{1} & \vec{n}-\vec{e}_{4}+\vec{e}_{2} & \vec{n}-\vec{e}_{4}+\vec{e}_{3} & \vec{n} & \vec{n}+\vec{e}_{1} & \vec{n}+\vec{e}_{2} \\
\vec{n}-\vec{e}_{4}+\vec{e}_{2} & \vec{n}-\vec{e}_{4}+\vec{e}_{3} & \vec{n} & \vec{n}+\vec{e}_{1} & \vec{n}+\vec{e}_{2} & \vec{n}+\vec{e}_{3} \\
\vec{n}-\vec{e}_{4}+\vec{e}_{3} & \vec{n} & \vec{n}+\vec{e}_{1} & \vec{n}+\vec{e}_{2} & \vec{n}+\vec{e}_{3} & \vec{n}+\vec{e}_{4}
\end{array}
$$

Целью настоящей работы является получение явного вида этих четырех рекуррентных соотношений.

\section{2. Нахождение многочленов \\ с недиагональными индексами}

Для получения рекуррентных соотношений, связывающих индексы (7), нам необходимо знать явный вид всех многочленов последовательности (6). Многочлены с индексами $\left[n_{1}, n_{2}\right]$ определяются формулой Родрига (5). Явное выражение для многочленов с индексами $\left(n_{1}, n_{1}-1, n_{2}, n_{2}\right)$ и $\left(n_{1}, n_{1}, n_{2}, n_{2}-1\right)$ получим с помощью следующего приема (см. [5]), позволяющего обобщить формулу Родрига на случай векторных индексов такого вида. Представим $Q_{\left(n_{1}, n_{1}-1, n_{2}, n_{2}\right)}$ как

$$
\begin{array}{r}
Q_{\left(n_{1}, n_{1}-1, n_{2}, n_{2}\right)}^{\left(\alpha_{1}, \alpha_{2}, \alpha\right)}(z)=w_{2}^{-1}(z) \frac{d^{n_{2}}}{d z^{n_{2}}}\left[w_{2}(z) z^{n_{2}} w_{1}^{-1}(z)\right. \\
\left.\times \frac{d^{n_{1}}}{d z^{n_{1}}}\left[w_{1}(z) z^{n_{1}}(1-z)^{\left(n_{1}+n_{2}\right)}\left(z-t\left(n_{1}, n_{2}\right)\right)\right]\right],
\end{array}
$$

где $t\left(n_{1}, n_{2}\right)$ - неизвестная константа. Из этого соотношения следует, что

$$
Q_{\left(n_{1}, n_{1}-1, n_{2}, n_{2}\right)}^{\left(\alpha_{1}, \alpha_{2}, \beta\right)}(z)=z Q_{\left(n_{1}-1, n_{1}-1, n_{2}, n_{2}\right)}^{\left(\alpha_{1}+1, \alpha_{2}+1, \alpha, \beta\right)}(z)-t Q_{\left(n_{1}-1, n_{1}-1, n_{2}, n_{2}\right)}^{\left(\alpha_{1}, \alpha_{2}, \alpha, \beta\right)}(z) .
$$

Правая часть (8) удовлетворяет $2 n_{1}+2 n_{2}-2$ соотношениям ортогональности. Тогда $t\left(n_{1}, n_{2}\right)$ находим из последнего соотношения ортогональности, которому должен удовлетворять многочлен 
$Q_{\left(n_{1}, n_{1}-1, n_{2}, n_{2}\right)}^{\left(\alpha_{1}, \alpha_{2}, \alpha\right)}(z)$

$$
\int_{0}^{1} Q_{\left(n_{1}, n_{1}-1, n_{2}, n_{2}\right)}^{\left(\alpha_{1}, \alpha_{2}, \beta\right)}(x) x^{n_{1}-1} w_{1}(x) d x=0 .
$$

Получаем, что

$$
t\left(n_{1}, n_{2}\right)=\frac{\int_{0}^{1} x^{n_{1}+\alpha_{1}}(1-x)^{\left(n_{1}+n_{2}+\alpha-1\right)} e^{\beta x} d x}{\int_{0}^{1} x^{n_{1}+\alpha_{1}-1}(1-x)^{\left(n_{1}+n_{2}+\alpha-1\right)} e^{\beta x} d x} .
$$

Явный вид для $Q_{\left(n_{1}, n_{1}, n_{2}, n_{2}-1\right)}^{\left(\alpha_{1}, \alpha_{2}, \alpha, \beta\right)}(z)$ следует из соотношения симметрии

$$
Q_{\left(n_{1}, n_{1}, n_{2}, n_{2}-1\right)}^{\left(\alpha_{1}, \alpha_{2}, \beta\right)}(z)=Q_{\left(n_{2}, n_{2}-1, n_{1}, n_{1}-1\right)}^{\left(\alpha_{2}, \alpha_{1}, \alpha, \beta\right)}(z) .
$$

Нас особенно будет интересовать случай

$$
\alpha_{1}=\alpha_{2}=0, \quad \alpha=1, \quad \beta=-1 \text {. }
$$

Для вычисления отношений интегралов (9) в этом случае мы получим следующие рекуррентные соотношения:

УтвеРЖДЕНИЕ 1. Для $t(n+1, n)$ u $t(n, n)$ в случае (11) сnраведливо

$$
\begin{aligned}
t(n+1, n) & =(3 n+2)-\frac{2 n+1}{-(3 n+1)+n[t(n, n)]^{-1}}, \\
t(n, n) & =3 n-\frac{2 n}{1-t(n, n-1)} .
\end{aligned}
$$

ДокАЗАТЕЛЬСтво. Найдем сначала рекуррентные соотношения для интегралов $J(n, m)$, участвующих в определении (9) констант $t\left(n_{1}, n_{2}\right)$ :

$$
J(n, m):=\int_{0}^{1} x^{n}(1-x)^{m} e^{-x} d x .
$$

Для этого рассмотрим аппроксимации Паде $\frac{P_{n, m}(z)}{Q_{n, m}(z)}$ к функции $e^{z}$ :

$$
Q_{n, m}(z) e^{z}-P_{n, m}(z)=R_{n, m}(z)=A_{n, m} z^{n+m+1}+\cdots .
$$


Интегральные представления для аппроксимаций Паде получаются из следующего тождества:

$$
\begin{gathered}
{\left[z^{n+m+1} \int_{0}^{\infty} t^{n}(1-t)^{m} e^{-z t} d t\right] e^{z}} \\
\quad-e^{z} z^{n+m+1} \int_{1}^{\infty} t^{n}(1-t)^{m} e^{-z t} d t \\
=e^{z} z^{n+m+1} \int_{0}^{1} t^{n}(1-t)^{m} e^{-z t} d t,
\end{gathered}
$$

откуда, в частности,

$$
\begin{aligned}
R_{n, m}(z) & =e^{z} z^{n+m+1} \int_{0}^{1} t^{n}(1-t)^{m} e^{-z t} d t \\
A_{n, m} & =\int_{0}^{1} t^{n}(1-t)^{m} d t .
\end{aligned}
$$

Тогда

$$
J(n, m)=R_{n, m}(1) e^{-1} .
$$

Известно (см., например, [6]), что функции $R_{n, m}(z)$ удовлетворяют следующим рекуррентным ссоотношениям:

$$
\begin{aligned}
& R_{n, m}(z)=R_{n-1, m}(z)-\frac{A_{n-1, m}}{A_{n-1, m-1}} z R_{n-1, m-1}, \\
& R_{n, m}(z)=R_{n, m-1}(z)-\frac{A_{n, m-1}}{A_{n-1, m-1}} z R_{n-1, m-1} .
\end{aligned}
$$

Отсюда, с учетом явного вида (13) констант $A_{n, m}$ и соотношения (14), получаем

$$
\begin{aligned}
& J(n, m)=-(n+m) J(n, m-1)+n J(n-1, m-1), \\
& J(n, m)=(n+m) J(n-1, m)-m J(n-1, m-1) .
\end{aligned}
$$

Учитывая, что

$$
t(n, n)=\frac{J(n, 2 n)}{J(n-1,2 n)}, \quad t(n+1, n)=\frac{J(n+1,2 n+1)}{J(n, 2 n+1)}
$$

и рекуррентные связи (15), получаем искомые уже нелинейные соотношения (12). 


\section{3. Нахождение коэффициентов рекуррентных соотношений}

Сначала мы получим коэффициенты рекуррентных соотношений для интересующего нас специального случая (11). Справедлива

Теорема 1'. Последовательность (6) многочленов $Q$, определяемых формулами (5), (8)-(10) (при $\left.\alpha_{1}=\alpha_{2}=0, \alpha=1, \beta=-1\right)$ удовлетворяет следующей системе рекуррентных соотношений

$$
\begin{aligned}
& Q_{(n+1, n, n, n)}=\left(z+A_{0}^{1}\right) Q_{(n, n, n, n)}+A_{1}^{1} Q_{(n, n, n, n-1)} \\
& +A_{2}^{1} Q_{(n, n, n-1, n-1)}+A_{3}^{1} Q_{(n, n-1, n-1, n-1)} \\
& +A_{4}^{1} Q_{(n-1, n-1, n-1, n-1)}, \\
& Q_{(n+1, n+1, n, n)}=\left(z+A_{0}^{2}\right) Q_{(n+1, n, n, n)}+A_{1}^{2} Q_{(n, n, n, n)} \\
& +A_{2}^{2} Q_{(n, n, n, n-1)}+A_{3}^{2} Q_{(n, n, n-1, n-1)}+A_{4}^{2} Q_{(n, n-1, n-1, n-1)}, \\
& Q_{(n+1, n+1, n+1, n)}=\left(z+A_{0}^{3}\right) Q_{(n+1, n+1, n, n)}+A_{1}^{3} Q_{(n+1, n, n, n)} \\
& +A_{2}^{3} Q_{(n, n, n, n)}+A_{3}^{3} Q_{(n, n, n, n-1)}+A_{4}^{3} Q_{(n, n, n-1, n-1)}, \\
& Q_{(n+1, n+1, n+1, n+1)}=\left(z+A_{0}^{4}\right) Q_{(n+1, n+1, n+1, n)}+A_{1}^{4} Q_{(n+1, n+1, n, n)} \\
& +A_{2}^{4} Q_{(n+1, n, n, n)}+A_{3}^{4} Q_{(n, n, n, n)}+A_{4}^{4} Q_{(n, n, n, n-1)},
\end{aligned}
$$

где для коэффициентов $A_{j}^{i}$ справедливо

$$
\begin{aligned}
& \left\{\begin{array}{l}
A_{0}^{1}=-t(n+1, n)-2 n, \\
A_{1}^{1}=-2-7 n, \\
A_{2}^{1}=(-7 n-2) t(n, n)-3 n^{2}+2 n, \\
A_{3}^{1}=-9 n^{2}-2 n, \\
A_{4}^{1}=\left(-9 n^{2}-2 n\right) t(n, n-1)+3 n^{2},
\end{array}\right. \\
& \left\{\begin{aligned}
& A_{0}^{2}=-5 n-4+t(n+1, n), \\
& A_{1}^{2}= t(n+1, n)^{2}+(-3 n-4) t(n+1, n) \\
&-7 n^{2}-3 n+1, \\
& A_{2}^{2}=\left(7 n^{2}+2 n\right) t(n+1, n)-24 n^{3}-25 n^{2}-6 n, \\
& A_{3}^{2}=\left(-24 n^{3}-25 n^{2}-6 n\right. \\
&\left.+\left(7 n^{2}+2 n\right) t(n+1, n)\right) t(n, n) \\
&+\left(3 n^{3}-2 n^{2}\right) t(n+1, n)-9 n^{4}-n^{3}+3 n^{2}, \\
& A_{4}^{2}=\left(9 n^{4}+2 n^{3}\right) t(n+1, n)-27 n^{5}-30 n^{4}-7 n^{3},
\end{aligned}\right.
\end{aligned}
$$




$$
\begin{aligned}
& \left\{\begin{array}{l}
A_{0}^{3}=-t(n+1, n+1)-2 n-1, \\
A_{1}^{3}=-7 n^{2}-9 n-3, \\
A_{2}^{3}=\left(-7 n^{2}-9 n-3\right) t(n+1, n)-3 n^{3}+n^{2}+3 n+1, \\
A_{3}^{3}=-9 n^{4}-8 n^{3}-2 n^{2} \\
A_{4}^{3}=\left(-9 n^{4}-8 n^{3}-2 n^{2}\right) t(n, n)+3 n^{4}+n^{3}
\end{array}\right. \\
& \left\{\begin{aligned}
A_{0}^{4}= & t(n+1, n+1)-5 n-6 \\
A_{1}^{4}= & t(n+1, n+1)^{2}+(-3 n-5) t(n+1, n+1) \\
& -3-7 n^{2}-10 n \\
A_{2}^{4}= & \left(7 n^{2}+9 n+3\right) t(n+1, n+1)-24 n^{3} \\
& -61 n^{2}-51 n-14 \\
A_{3}^{4}= & \left(\left(7 n^{2} e+9 n+3\right) t(n+1, n+1)-24 n^{3}\right. \\
& \left.-61 n^{2}-51 n-14\right) t(n+1, n) \\
& +\left(3 n^{3}-n^{2}-3 n-1\right) t(n+1, n+1) \\
& -9 n^{4}-7 n^{3}+13 n^{2}+15 n+4 \\
A_{4}^{4}= & \left(9 n^{4}+8 n^{3}+2 n^{2}\right) t(n+1, n+1) \\
& -27 n^{5}-57 n^{4}-38 n^{3}-8 n^{2}
\end{aligned}\right.
\end{aligned}
$$

ДокАзАТЕЛЬСтво. Для нахождения коэффициентов $A_{j}^{i}$ воспользуемся явным видом многочленов $Q_{[n, m]}(z)$, который получается из формулы Родрига (5) путем последовательного применения формулы Лейбница для дифференцирования произведения функций и бинома Ньютона:

$$
\begin{aligned}
Q\left[n_{1}, n_{2}\right]=\sum_{\nu=0}^{2\left(n_{1}+n_{2}\right)} \sum_{k=\max \left(0, n_{1}+n_{2}-\nu\right)}^{\min \left(2\left(n_{1}+n_{2}\right)-\nu, n_{1}+n_{2}\right)} \sum_{j=0}^{\min \left(n_{2}, k\right)} \times \\
\quad \times \quad \sum_{m=0}^{\min \left(2\left(n_{1}+n_{2}\right)-\nu-k, n_{1}+n_{2}-k\right)}(-1)^{\nu}\left(\begin{array}{c}
n_{2} \\
j
\end{array}\right)\left(\begin{array}{c}
n_{1}+n_{2}-j \\
k-j
\end{array}\right) \times \\
\quad \times\left(\begin{array}{c}
n_{1}+n_{2}-m \\
2\left(n_{1}+n_{2}\right)-\nu-m-k
\end{array}\right)\left(\begin{array}{c}
n_{1}+n_{2}-k \\
m
\end{array}\right) \times \\
\quad \times\left(n_{1}\right)_{k-j}\left(n_{2}\right)_{j}\left(n_{1}+n_{2}+1\right)_{m} z^{\nu} .
\end{aligned}
$$

Отсюда получаем коэффициенты $c_{p}\left[n_{1}, n_{2}\right]$ при степенях $z^{2\left(n_{1}+n_{2}\right)-p}, p<n_{1}+n_{2}, p<n_{2}$. При $n_{1}=n, n_{2}=n+k$ имеeM

$$
c_{0}[n, n+k]=1,
$$




$$
\begin{aligned}
& c_{1}[n, n+k]=-7 n^{2}+(-7 k-4) n-2 k^{2}-2 k, \\
& c_{2}[n, n+k]=\frac{49}{2} n^{4}+(9+49 k) n^{3}+\frac{1}{2}\left(-3+77 k^{2}+27 k\right) n^{2} \\
& +\left(-3+\frac{13}{2} k^{2}-\frac{3}{2} k+14 k^{3}\right) n+\frac{1}{2} k\left(2 k^{2}+4 k^{3}-k-3\right), \\
& c_{3}[n, n+k]=-\frac{343}{6} n^{6}+\left(-\frac{343}{2} k+35\right) n^{5} \\
& \quad+\frac{1}{2}\left(175 k+\frac{11}{3}-441 k^{2}\right) n^{4} \\
& \quad+\left(\frac{11}{3} k+\frac{29}{3}-\frac{931}{6} k^{3}+94 k^{2}\right) n^{3} \\
& \quad+\left(-63 k^{4}+\frac{29}{2} k+\frac{107}{2} k^{3}+k^{2}-\frac{2}{3}\right) n^{2} \\
& \quad+\left(16 k^{4}-\frac{5}{6} k^{3}-\frac{8}{3}+\frac{15}{2} k^{2}-\frac{2}{3} k-14 k^{5}\right) n \\
& \quad-\frac{1}{3} k(k-1)\left(4 k^{4}-2 k^{3}-k^{2}-5 k-4\right), \\
& c_{4}[n, n+k]=\frac{2401}{24} n^{8}+\frac{1}{6}(-1421+2401 k) n^{7}
\end{aligned}
$$$$
+\frac{1}{12}\left(-9947 k+2453+8575 k^{2}\right) n^{6}
$$$$
+\frac{1}{12}\left(-15393 k^{2}+8918 k^{3}+7359 k-1218\right) n^{5}
$$$$
+\frac{1}{24}\left(11809 k^{4}-27230 k^{3}-6090 k+19387 k^{2}+729\right) n^{4}
$$$$
+\frac{1}{12}\left(2548 k^{5}-7421 k^{4}+7122 k^{3}-3296 k^{2}+729 k+88\right) n^{3}
$$$$
+\frac{1}{24}\left(1400 k^{6}-4980 k^{5}+6121 k^{4}\right.
$$$$
\left.-3798 k^{3}+1229 k^{2}+264 k-8\right) n^{2}
$$$$
+\frac{1}{6}\left(56 k^{7}-238 k^{6}+363 k^{5}-287 k^{4}\right.
$$$$
\left.+125 k^{3}+29 k^{2}-2 k-15\right) n
$$$$
+\frac{1}{24} k(k-1)\left(16 k^{6}-64 k^{5}+84 k^{4}-60 k^{3}+21 k^{2}+35 k+30\right) \text {, }
$$

$c_{5}[n, n+k]=-\frac{16807}{120} n^{10}+\left(686-\frac{16807}{24} k\right) n^{9}$ 


$$
\begin{aligned}
& +\left(-\frac{4802}{3} k^{2}+3087 k-\frac{16681}{12}\right) n^{8} \\
& +\frac{1}{12}\left(-26411 k^{3}+75656 k^{2}-66724 k+18382\right) n^{7} \\
& +\frac{1}{120}\left(-241815 k^{4}+919240 k^{3}-1201715 k^{2}\right. \\
& +643370 k-119251) n^{6} \\
& +\frac{1}{120}\left(-154007 k^{5}+731640 k^{4}-1269805 k^{3}\right. \\
& \left.+1004650 k^{2}-357753 k+41948\right) n^{5} \\
& +\frac{1}{24}\left(-13818 k^{6}+79072 k^{5}-171897 k^{4}+180640 k^{3}\right. \\
& \left.-94739 k^{2}+20974 k-1224\right) n^{4} \\
& +\frac{1}{24}\left(-4312 k^{7}+29000 k^{6}-76215 k^{5}+100598 k^{4}\right. \\
& \left.-70227 k^{3}+22988 k^{2}-2448 k+228\right) n^{3} \\
& +\frac{1}{24}\left(-896 k^{8}+6960 k^{7}-21594 k^{6}+34594 k^{5}-30489 k^{4}\right. \\
& \left.+13508 k^{3}-2229 k^{2}+342 k+\frac{48}{5}\right) n^{2} \\
& +\frac{1}{120}\left(-560 k^{9}+4960 k^{8}-17860 k^{7}+33920 k^{6}-36503 k^{5}\right. \\
& \left.+20870 k^{4}-5025 k^{3}+1030 k^{2}+48 k-288\right) n \\
& -\frac{1}{60} k(k-1)(k-2)\left(16 k^{7}-112 k^{6}+292 k^{5}-360 k^{4}\right. \\
& \left.+209 k^{3}+10 k^{2}+57 k+36\right) .
\end{aligned}
$$

Аналогично для коэффициентов $\tilde{c}_{p}[n, n+k]$ полиномов $Q[n, n+k]$ из (5) с параметрами $\alpha_{1}=\alpha_{2}=1, \alpha=-\beta=1$ имеем $\tilde{c}_{0}[n, n+k]=1$, $\tilde{c}_{1}[n, n+k]=-7 n^{2}+(-6-7 k) n-3 k-2 k^{2}$, $\tilde{c}_{2}[n, n+k]=\frac{49}{2} n^{4}+(49 k+23) n^{3}+\frac{1}{2}\left(77 k^{2}+69 k-1\right) n^{2}$ $+\left(14 k^{3}+\frac{35}{2} k^{2}-\frac{1}{2} k-5\right) n+\frac{1}{2} k\left(-k-5+6 k^{2}+4 k^{3}\right)$, $\tilde{c}_{3}[n, n+k]=-\frac{343}{6} n^{6}+\left(-\frac{343}{2} k-14\right) n^{5}$ 


$$
\begin{aligned}
& +\left(-\frac{441}{2} k^{2}-35 k+\frac{197}{6}\right) n^{4} \\
& +\frac{1}{6}\left(-931 k^{3}-192 k^{2}+394 k+100\right) n^{3} \\
& +\left(-63 k^{4}-13 k^{3}+\frac{103}{2} k^{2}+25 k-\frac{5}{3}\right) n^{2} \\
& +\left(-14 k^{5}-2 k^{4}+\frac{56}{3} k^{3}+12 k^{2}-\frac{5}{3} k-\frac{14}{3}\right) n \\
& -\frac{1}{6} k(k-1)\left(8 k^{4}+8 k^{3}-8 k^{2}-19 k-14\right), \\
& \tilde{c}_{4}[n, n+k]=\frac{2401}{24} n^{8}+\frac{1}{6}(2401 k-735) n^{7} \\
& +\frac{1}{12}\left(8575 k^{2}-5145 k-445\right) n^{6} \\
& +\frac{1}{12}\left(8918 k^{3}-8043 k^{2}-1335 k+586\right) n^{5} \\
& +\frac{1}{24}\left(11809 k^{4}-14490 k^{3}-3101 k^{2}+2930 k+157\right) n^{4} \\
& +\frac{1}{12}\left(2548 k^{5}-4047 k^{4}-876 k^{3}+1532 k^{2}+157 k+152\right) n^{3} \\
& +\frac{1}{24}\left(1400 k^{6}-2796 k^{5}-467 k^{4}+1666 k^{3}+197 k^{2}+456 k\right) n^{2} \\
& +\frac{1}{6}\left(56 k^{7}-138 k^{6}-9 k^{5}+118 k^{4}+10 k^{3}+55 k^{2}-27\right) n \\
& +\frac{1}{24} k(k-1)\left(16 k^{6}-32 k^{5}-28 k^{4}+28 k^{3}+29 k^{2}+63 k+54\right), \\
& \tilde{c}_{5}[n, n+k]=-\frac{16807}{120} n^{10}+\frac{1}{24}(-16807 k+11662) n^{9} \\
& +\frac{1}{24}\left(-38416 k^{2}+52479 k-12390\right) n^{8} \\
& +\frac{1}{12}\left(-26411 k^{3}+53704 k^{2}-24780 k+736\right) n^{7} \\
& +\frac{1}{120}\left(-241815 k^{4}+655130 k^{3}-451385 k^{2}+25760 k+23389\right) n^{6} \\
& +\frac{1}{120}\left(-154007 k^{5}+524370 k^{4}-486855 k^{3}+47970 k^{2}\right. \\
& +70167 k-16022) n^{5}+\frac{1}{24}\left(-13818 k^{6}+57071 k^{5}\right. \\
& \left.-67747 k^{4}+11105 k^{3}+17683 k^{2}-8011 k+772\right) n^{4}
\end{aligned}
$$




$$
\begin{aligned}
& +\frac{1}{24}\left(-4312 k^{7}+21104 k^{6}-31029 k^{5}+7996 k^{4}+11977 k^{3}\right. \\
& \left.-8432 k^{2}+1544 k+532\right) n^{3} \\
& +\frac{1}{120}\left(-4480 k^{8}+25560 k^{7}-45550 k^{6}\right. \\
& \left.+17325 k^{5}+23005 k^{4}-23185 k^{3}+6395 k^{2}+3990 k-272\right) n^{2} \\
& +\frac{1}{120}\left(-560 k^{9}+3680 k^{8}-7820 k^{7}+4140 k^{6}+4757 k^{5}-6600 k^{4}\right. \\
& \left.+2535 k^{3}+1960 k^{2}-272 k-528\right) n \\
& -\frac{1}{120} k(k-1)(k-2)\left(32 k^{7}-144 k^{6}+104 k^{5}+180 k^{4}-82 k^{3}\right. \\
& \left.+165 k^{2}+259 k+132\right) .
\end{aligned}
$$

Приравнивая последовательно к нулю коэффициенты при $z^{4 n}$, $z^{4 n-1}, z^{4 n-2}, z^{4 n-3}$ и $z^{4 n-4}$ в первом из соотношений (16), получим соответственно

$$
\begin{aligned}
& c_{0}[n, n] A_{0}^{1}=-t(n+1, n) c_{0}[n, n]-c_{1}[n, n]+\tilde{c}_{1}[n, n], \\
& \tilde{c}_{0}[n, n-1] A_{1}^{1}=-t(n+1, n) c_{1}[n, n]-c_{2}[n, n]+\tilde{c}_{2}[n, n]-A_{0}^{1} c_{1}[n, n], \\
& c_{0}[n, n-1] A_{2}^{1}=-t(n+1, n) c_{2}[n, n]-c_{3}[n, n]+\tilde{c}_{3}[n, n]-A_{0}^{1} c_{2}[n, n] \\
& \quad+A_{1}^{1} t(n, n) c_{0}[n, n-1]-A_{1}^{1} \tilde{c}_{1}[n, n-1], \\
& \quad \tilde{c}_{0}[n-1, n-1] A_{3}^{1}=-t(n+1, n) c_{3}[n, n]-c_{4}[n, n] \\
& \quad+\tilde{c}_{4}[n, n]-A_{0}^{1} c_{3}[n, n]+A_{1}^{1} t(n, n) c_{1}[n, n-1] \\
& \quad-A_{1}^{1} \tilde{c}_{2}[n, n-1]-A_{2}^{1} c_{1}[n, n-1], \\
& \quad c_{0}[n, n] A_{4}^{1}=-t\left(n+1, n c_{4}[n, n]-c_{5}[n, n]+\tilde{c}_{5}[n, n]-A_{0}^{1} c_{4}[n, n]\right. \\
& \quad+A_{1}^{1} t(n, n) c_{2}[n, n-1]-A_{1}^{1} \tilde{c}_{3}[n, n-1]-A_{2}^{1} c_{2}[n, n-1] \\
& \quad+A_{3}^{1} t(n, n-1) c_{0}[n-1, n-1]-A_{3}^{1} \tilde{c}_{1}[n-1, n-1] .
\end{aligned}
$$

Подставляя в полученные выражения явный вид $c_{p}$ и $\tilde{c}_{p}$ из (19.1) и (19.2), приходим к формулам (17.1) для коэффициентов первого рекуррентного соотношения из (16). Коэффициенты остальных рекуррентных соотношений системы (16) получаются таким же образом.

Теорема доказана.

Аналогично получаются коэффициенты рекуррентных соотношений и в общем случае (при произвольных значениях параметров $\left.\alpha_{1}, \alpha_{2}, \alpha, \beta\right)$. 


\section{Справедлива}

Теорема 1. Знаменатели аппроксимаций Эрмита-Паде (со стариим коэфбициентом 1) для системы функиий (2) удовлетворяют системе рекуррентных соотношений (16), где для коэффициентов $A_{j}^{i}$ справедливо

$$
\begin{aligned}
& \beta A_{0}^{1}=-t(n+1, n) \beta+2 n, \\
& \beta^{2} A_{1}^{1}=-n\left(\alpha_{1}+\alpha_{2}+2 \alpha+7 n\right), \\
& \beta^{3} A_{2}^{1}=\left(-7 n^{2} \beta+\left(-\alpha_{1} \beta-\alpha_{2} \beta-2 \alpha \beta\right) n\right) t(n, n)+3 n^{3}+\left(4 \alpha_{1}+\alpha\right. \\
& \left.+3 \beta-3 \alpha_{2}\right) n^{2}+\left(\alpha_{1}^{2}-\alpha_{2} \alpha+\alpha_{1} \beta+\alpha_{1} \alpha+\alpha_{2} \beta-\alpha_{1} \alpha_{2}\right) n, \\
& \beta^{4} A_{3}^{1}=9 n^{4}+\left(9 \alpha_{2}-6 \alpha+3-\beta-15 \alpha_{1}\right) n^{3}+\left(-7 \alpha_{1}^{2}+6 \alpha_{2} \alpha\right. \\
& \left.+4 \alpha_{1}-8 \alpha_{1} \alpha-3 \alpha_{2}-2 \alpha_{1} \beta+\alpha_{2} \beta+6 \alpha_{1} \alpha_{2}+\alpha-\alpha^{2}\right) n^{2} \\
& +\left(\alpha_{1} \alpha+\alpha_{1} \alpha_{2} \beta-\alpha_{1} \alpha 2-2 \alpha_{1}^{2} \alpha-\alpha_{1} \alpha^{2}-\alpha_{1}^{3}\right. \\
& \left.+\alpha_{1}^{2} \alpha_{2}+\alpha_{2} \alpha^{2}-\alpha_{1}^{2} \beta+2 \alpha_{1} \alpha_{2} \alpha+\alpha_{1}^{2}-\alpha_{2} \alpha\right) n, \\
& \beta^{4} A_{4}^{1}=\left(-9 n^{4}-\left(-3+15 \alpha_{1}-9 \alpha_{2}+6 \alpha+\beta\right) n^{3}\right. \\
& -\left(-\alpha+\alpha^{2}-4 \alpha_{1}+3 \alpha_{2}-6 \alpha_{1} \alpha_{2}\right. \\
& \left.-\alpha_{2} \beta+2 \alpha_{1} \beta+7 \alpha_{1}^{2}-6 \alpha_{2} \alpha+8 \alpha_{1} \alpha\right) n^{2} \\
& -\left(\alpha_{1} \alpha^{2}-\alpha_{1}^{2} \alpha_{2}+\alpha_{1} \alpha_{2}-\alpha_{2} \alpha^{2}-\alpha_{1} \alpha_{2} \beta-\alpha_{1}^{2}\right. \\
& \left.\left.+2 \alpha_{1}^{2} \alpha+\alpha_{2} \alpha+\alpha_{1}^{3}-\alpha_{1} \alpha-2 \alpha_{1} \alpha_{2} \alpha+\alpha_{1}^{2} \beta\right) n\right) t(n, n-1) \\
& +3 n^{4}-\left(-7 \alpha_{1}-\alpha-\beta+3 \alpha_{2}\right) n^{3} \\
& -\left(\alpha_{2} \beta-5 \alpha_{1}^{2}+\alpha_{2} \alpha-2 \alpha_{1} \beta+4 \alpha_{1} \alpha_{2}-2 \alpha_{1} \alpha\right) n^{2} \\
& -\left(-\alpha_{1}^{2} \alpha+\alpha_{1}^{2} \alpha_{2}+\alpha_{1} \alpha_{2} \alpha+\alpha_{1} \alpha_{2} \beta-\alpha_{1}^{3}-\alpha_{1}^{2} \beta\right) n, \\
& \beta A_{0}^{2}=-\beta+5 n+2+\alpha+\alpha_{1}+t(n+1, n) \beta, \\
& \beta^{2} A_{1}^{2}=t(n+1, n)^{2} \beta^{2}+\left(-\beta^{2}+2 \beta+3 n \beta+\alpha \beta+\alpha_{1} \beta\right) t(n+1, n) \\
& -7 n^{2}+\left(-\alpha_{1}-\alpha_{2}-2-2 \alpha-\beta\right) n-\alpha_{1} \beta-\beta, \\
& \beta^{3} A_{2}^{2}=\left(7 n^{2} \beta+\left(\alpha_{1} \beta+\alpha_{2} \beta+2 \alpha \beta\right) n\right) t(n+1, n)+24 n^{3} \\
& +\left(7 \alpha_{2}+7 \alpha_{1}-4 \beta+7+14 \alpha\right) n^{2} \\
& +\left(\alpha_{1}+\alpha_{2}+2 \alpha+2 \alpha^{2}+\alpha_{1}^{2}+2 \alpha_{1} \alpha+\alpha_{2}^{2}+2 \alpha_{2} \alpha-2 \alpha \beta\right) n, \\
& \beta^{4} A_{3}^{2}=\left(-3 n^{3} \beta+\left(-\alpha \beta-4 \alpha_{1} \beta+3 \alpha_{2} \beta-3 \beta^{2}+7 t(n, n) \beta^{2}\right) n^{2}\right. \\
& +\left(\alpha_{1} \alpha_{2} \beta+t(n, n) \beta^{2} \alpha_{1}+t(n, n) \beta^{2} \alpha_{2}+2 t(n, n) \beta^{2} \alpha\right. \\
& \left.\left.-\alpha_{1}^{2} \beta-\beta^{2} \alpha_{2}-\alpha_{1} \alpha \beta+\alpha_{2} \alpha \beta-\alpha_{1} \beta^{2}\right) n\right) t(n+1, n)
\end{aligned}
$$




$$
\begin{aligned}
& -9 n^{4}+\left(9 \alpha_{2}-8 \beta-3+24 t(n, n) \beta-15 \alpha_{1}-6 \alpha\right) n^{3} \\
& +\left(-4 \alpha_{1}+6 \alpha_{1} \alpha_{2}+6 \alpha_{2} \alpha-4 t(n, n) \beta^{2}+7 t(n, n) \beta \alpha_{1}\right. \\
& +14 t(n, n) \beta \alpha-\alpha^{2}+7 t(n, n) \beta \alpha_{2}-7 \alpha_{1}^{2}-2 \alpha \beta \\
& \left.-8 \alpha_{1} \alpha+7 t(n, n) \beta+3 \alpha_{2}-3 \beta-3 \alpha_{1} \beta-7 \alpha_{2} \beta-\alpha\right) n^{2} \\
& +\left(-2 \alpha_{1}^{2} \alpha-\alpha_{1}^{2}+2 t(n, n) e \beta \alpha^{2}-2 t(n, n) \beta^{2} \alpha\right. \\
& +t(n, n) \beta \alpha_{1}+t(n, n) \beta \alpha_{2}-\alpha_{1} \alpha+\alpha_{2} \alpha-\alpha_{1} \alpha^{2}-\alpha_{1}^{2} \beta \\
& +2 t(n, n) \beta \alpha_{1} \alpha+2 t(n, n) \beta \alpha_{2} \alpha-\alpha_{1} \beta+\alpha_{1} \alpha_{2}-\alpha_{2} \beta \\
& +t(n, n) \beta \alpha_{1}^{2}+t(n, n) \beta \alpha_{2}^{2}+\alpha_{2} \alpha^{2}-\alpha_{1}^{3}+2 t(n, n) \beta \alpha \\
& \left.-\alpha_{2}^{2} \beta+2 \alpha_{1} \alpha_{2} \alpha+\alpha_{1}^{2} \alpha 2-2 \alpha_{2} \alpha \beta\right) n, \\
& \beta^{5} A_{4}^{2}=\left(9 n^{4} \beta+\left(-9 \alpha_{2} \beta+\beta^{2}+15 \alpha_{1} \beta-3 \beta+6 \alpha \beta\right) n^{3}\right. \\
& +\left(7 \alpha_{1}^{2} \beta-6 \alpha_{1} \alpha_{2} \beta+8 \alpha_{1} \alpha \beta+\alpha^{2} \beta\right. \\
& \left.-6 \alpha_{2} \alpha \beta-\alpha \beta+3 \alpha_{2} \beta-4 \alpha_{1} \beta+2 \alpha_{1} \beta^{2}-\beta^{2} \alpha_{2}\right) n^{2} \\
& +\left(\alpha_{2} \alpha \beta-\alpha_{1}^{2} \alpha_{2} \beta+\alpha_{1} \alpha_{2} \beta+\alpha_{1}^{3} \beta-\alpha_{1} \beta^{2} \alpha_{2}\right. \\
& -\alpha_{1} \alpha \beta+\beta^{2} \alpha_{1}^{2}-\alpha_{1}^{2} \beta-\alpha_{2} \alpha^{2} \beta+2 \alpha_{1}^{2} \alpha \beta \\
& \left.\left.+\alpha_{1} \alpha^{2} \beta-2 \alpha_{1} \alpha_{2} \alpha \beta\right) n\right) t(n+1, n)+27 n^{5} \\
& +\left(54 \alpha_{1}-27 \alpha_{2}+27 \alpha-3 \beta\right) n^{4}+\left(3 \beta-27 \alpha_{2} \alpha-27 \alpha_{1} \alpha_{2}\right. \\
& \left.+36 \alpha_{1}^{2}+3 \alpha_{2} \beta-\alpha_{1} \beta+9 \alpha^{2}-3+45 \alpha_{1} \alpha-4 \alpha \beta\right) n^{3} \\
& +\left(-4 \alpha_{1}+3 \alpha_{2}-\alpha-\alpha^{2} \beta+21 \alpha_{1}^{2} \alpha+\alpha^{3}+\alpha \beta-18 \alpha_{1} \alpha_{2} \alpha\right. \\
& +12 \alpha_{1} \alpha^{2}+3 \alpha_{1}^{2} \beta-9 \alpha_{1}^{2} \alpha_{2}+4 \alpha_{1} \beta \\
& \left.-2 \alpha_{1} \alpha_{2} \beta+10 \alpha_{1}^{3}+4 \alpha_{2} \alpha \beta-4 \alpha_{1} \alpha \beta-3 \alpha_{2} \beta-9 \alpha_{2} \alpha^{2}\right) n^{2} \\
& +\left(-\alpha_{1}^{2}-3 \alpha_{1} \alpha_{2} \alpha^{2}-\alpha_{2} \alpha \beta+\alpha_{1} \alpha \beta+\alpha_{1}^{4}-\alpha_{1} \alpha_{2} \beta\right. \\
& +\alpha_{1}^{2} \beta-3 \alpha_{1}^{2} \alpha_{2} \alpha+\alpha_{1} \alpha_{2}+3 \alpha_{1}^{3} \alpha-\alpha_{1}^{3} \alpha_{2}-\alpha_{1} \alpha+\alpha_{2} \alpha \\
& \left.+\alpha_{1}^{3} \beta-\alpha_{2} \alpha^{3}+3 \alpha_{1}^{2} \alpha^{2}-\alpha_{1} \alpha^{2} \beta+\alpha_{2} \alpha^{2} \beta-\alpha_{1}^{2} \alpha_{2} \beta+\alpha_{1} \alpha^{3}\right) n, \\
& \beta A_{0}^{3}=-t(n+1, n+1) \beta+2 n+1, \\
& \beta^{2} A_{1}^{3}=-\left(2+\alpha_{1}+\alpha+7 n+2 n \alpha+n \alpha_{1}+n \alpha_{2}+7 n^{2}\right), \\
& \beta^{3} A_{2}^{3}=\left(-7 n^{2} \beta+\left(-7 \beta-\alpha_{2} \beta-\alpha_{1} \beta-2 \alpha \beta\right) n\right. \\
& \left.-\alpha_{1} \beta-\alpha \beta-2 \beta\right) t(n+1, n)+3 n^{3} \\
& +\left(3 \beta+4 \alpha_{2}+1+\alpha-3 \alpha_{1}\right) n^{2}+\left(\alpha_{2} \beta-\alpha_{1}+\alpha_{1} \beta+3 \beta+\alpha_{2}^{2}\right. \\
& \left.+\alpha_{2}+\alpha_{2} \alpha-\alpha_{1} \alpha_{2}-\alpha_{1} \alpha\right) n+\beta+\alpha_{1} \beta \text {, }
\end{aligned}
$$




$$
\begin{aligned}
\beta^{4} A_{3}^{3} & =-9 n^{4}+\left(-3-15 \alpha_{2}-6 \alpha-\beta+9 \alpha_{1}\right) n^{3} \\
& +\left(-\alpha+6 \alpha_{1} \alpha_{2}+3 \alpha_{1}-2 \alpha_{2} \beta-4 \alpha_{2}-\alpha^{2}-7 \alpha_{2}^{2}-8 \alpha_{2} \alpha\right. \\
& \left.+\alpha_{1} \beta+6 \alpha_{1} \alpha\right) n^{2}+\left(-2 \alpha_{2}^{2} \alpha-\alpha_{2}^{2} \beta+2 \alpha_{1} \alpha_{2} \alpha+\alpha_{1} \alpha_{2} \beta\right. \\
& \left.-\alpha_{2}^{2}-\alpha_{2} \alpha+\alpha_{1} \alpha^{2}+\alpha_{1} \alpha+\alpha_{2}^{2} \alpha_{1}-\alpha_{2} \alpha^{2}-\alpha_{2}^{3}+\alpha_{1} \alpha_{2}\right) n, \\
\beta^{4} A_{4}^{3} & =\left(-9 n^{4}+\left(-3-15 \alpha_{2}-6 \alpha-\beta+9 \alpha_{1}\right) n^{3}\right. \\
& +\left(-\alpha+6 \alpha_{1} \alpha_{2}+3 \alpha_{1}-2 \alpha_{2} \beta-4 \alpha_{2}-\alpha^{2}-7 \alpha_{2}^{2}\right. \\
& \left.-8 \alpha_{2} \alpha+\alpha_{1} \beta+6 \alpha_{1} \alpha\right) n^{2}+\left(-2 \alpha_{2}^{2} \alpha-\alpha_{2}^{2} \beta+2 \alpha_{1} \alpha_{2} \alpha+\alpha_{1} \alpha_{2} \beta\right. \\
& \left.\left.-\alpha_{2}^{2}-\alpha_{2} \alpha+\alpha_{1} \alpha^{2}+\alpha_{1} \alpha+\alpha_{2}^{2} \alpha 1-\alpha_{2} \alpha^{2}-\alpha_{2}^{3}+\alpha_{1} \alpha_{2}\right) n\right) t(n, n) \\
& +3 n^{4}+\left(1+\beta+7 \alpha_{2}+\alpha-3 \alpha_{1}\right) n^{3} \\
& +\left(2 \alpha_{2}-\alpha_{1} \beta+2 \alpha_{2} \alpha+2 \alpha_{2} \beta-4 \alpha_{1} \alpha_{2}-\alpha_{1} \alpha-\alpha_{1}+5 \alpha 2^{2}\right) n^{2} \\
& +\left(\alpha_{2}^{2} \alpha+\alpha_{2}^{2}+\alpha_{2}^{3}+\alpha_{2}^{2} \beta-\alpha_{1} \alpha_{2}-\alpha_{1} \alpha_{2} \beta-\alpha_{1} \alpha_{2} \alpha-\alpha_{2}^{2} \alpha_{1}\right) n,
\end{aligned}
$$$$
\beta A_{0}^{4}=-\beta+5 n+4+\alpha+\alpha_{2}+t(n+1, n+1) \beta,
$$$$
\beta^{2} A_{1}^{4}=t(n+1, n+1)^{2} \beta^{2}
$$$$
+\left(3 n \beta+\alpha \beta-\beta^{2}+3 \beta+\alpha_{2} \beta\right) t(n+1, n+1)-7 n^{2}
$$$$
+\left(-\beta-\alpha_{1}-9-2 \alpha-\alpha_{2}\right) n-\beta-3-\alpha_{1}-\alpha-\alpha_{2} \beta \text {, }
$$$$
\beta^{3} A_{2}^{4}=\left(2 \beta+\left(7 \beta+\alpha_{1} \beta+\alpha_{2} \beta+2 \alpha \beta\right) n\right.
$$$$
\left.+\alpha \beta+\alpha_{1} \beta+7 n^{2} \beta\right) t(n+1, n+1)+2 n^{3}
$$$$
+\left(43+7 \alpha_{2}+14 \alpha+7 \alpha_{1}-4 \beta\right) n^{2}-\alpha \beta+\left(27+\alpha_{2}^{2}+4 \alpha_{2}+12 \alpha_{1}\right.
$$$$
\left.+16 \alpha+2 \alpha_{1} \alpha+2 \alpha_{2} \alpha+2 \alpha^{2}+\alpha_{1}^{2}-2 \alpha \beta-4 \beta\right) n-\beta+6+5 \alpha_{1}
$$

$$
\begin{aligned}
& +5 \alpha+2 \alpha_{1} \alpha+\alpha^{2}+\alpha_{1}^{2}, \\
\beta^{4} A_{3}^{4} & =\left(\left(7 t(n+1, n) \beta^{2}+3 \alpha_{1} \beta-3 \beta^{2}-\alpha \beta-4 \alpha_{2} \beta-\beta\right) n^{2}\right. \\
& +t(n+1, n) \beta^{2} \alpha_{1}-3 n^{3} \beta-\beta^{2}+t(n+1, n) \beta^{2} \alpha \\
& +2 t(n+1, n) \beta^{2}+\left(\alpha_{1} \beta-\alpha_{2}^{2} \beta-3 \beta^{2}-\alpha_{2} \alpha \beta-\beta^{2} \alpha_{2}\right. \\
& +\alpha_{1} \alpha \beta+\alpha_{1} \alpha_{2} \beta+7 t(n+1, n) \beta^{2}-\alpha_{2} \beta+2 t(n+1, n) \beta^{2} \alpha \\
& \left.+t(n+1, n) \beta^{2} \alpha_{1}+t(n+1, n) \beta^{2} \alpha_{2}-\alpha_{1} \beta^{2}\right) n \\
& \left.-\alpha_{1} \beta^{2}\right) t(n+1, n+1)-9 n^{4} \\
& +\left(-9-6 \alpha-15 \alpha_{2}-8 \beta+9 \alpha_{1}+24 t(n+1, n) \beta\right) n^{3} \\
& +t(n+1, n) \beta \alpha_{1}^{2}+\left(-2 \alpha \beta-7 \alpha_{2}^{2}-12 \alpha_{2}-8 \alpha_{2} \alpha-3 \alpha\right. \\
& -4 t(n+1, n) \beta^{2}+14 t(n+1, n) \beta \alpha-7 \alpha_{1} \beta+6 \alpha_{1} \alpha
\end{aligned}
$$




$$
\begin{aligned}
& +7 t(n+1, n) \beta \alpha_{2}-17 \beta+7 t(n+1, n) \beta \alpha_{1}+43 t(n+1, n) \beta \\
& \left.-\alpha^{2}-2+6 \alpha_{1} \alpha_{2}+9 \alpha_{1}-3 \alpha_{2} \beta\right) n^{2}-t(n+1, n) \beta^{2} \alpha \\
& +\left(2 \alpha_{1}-2 \alpha_{2}-12 \beta-2 \alpha_{2}^{2} \alpha-3 \alpha_{2}^{2}+2 t(n+1, n) \beta \alpha_{2} \alpha\right. \\
& +3 \alpha_{1} \alpha-3 \alpha_{2} \alpha+\alpha_{1} \alpha^{2}+12 t(n+1, n) \beta \alpha_{1}+16 t(n+1, n) \beta \alpha \\
& -3 \alpha \beta+2 \alpha_{1} \alpha_{2} \alpha-\alpha_{1}^{2} \beta+4 t(n+1, n) \beta \alpha_{2}+t(n+1, n) \beta \alpha_{2}^{2} \\
& -\alpha_{2}^{2} \beta+27 t(n+1, n) \beta-10 \alpha_{1} \beta+3 \alpha_{1} \alpha_{2}-2 \alpha_{2} \beta \\
& -\alpha_{2} \alpha^{2}-\alpha_{2}^{3}+\alpha_{2}^{2} \alpha_{1}+2 t(n+1, n) \beta \alpha_{1} \alpha-2 \alpha_{1} \alpha \beta \\
& +t(n+1, n) \beta \alpha_{1}^{2}+2 t(n+1, n) \beta \alpha^{2}-2 t(n+1, n) \beta^{2} \alpha \\
& \left.-4 t(n+1, n) \beta^{2}\right) n--\alpha_{1} \alpha \beta-3 \beta+5 t(n+1, n) \beta \alpha_{1} \\
& -4 \alpha_{1} \beta-\alpha \beta+6 t(n+1, n) \beta+t(n+1, n) \beta \alpha^{2} \\
& +5 t(n+1, n) \beta \alpha-\alpha_{1}^{2} \beta-t(n+1, n) \beta^{2}+2 t(n+1, n) \beta \alpha_{1} \alpha, \\
& \beta^{5} A_{4}^{4}=\left(9 n^{4} \beta+\left(\beta^{2}+6 \alpha \beta+15 \alpha_{2} \beta+3 \beta-9 \alpha_{1} \beta\right) n^{3}\right. \\
& +\left(2 \beta^{2} \alpha_{2}-3 \alpha_{1} \beta+\alpha \beta-6 \alpha_{1} \alpha_{2} \beta+4 \alpha_{2} \beta-6 \alpha_{1} \alpha \beta+\alpha^{2} \beta\right. \\
& \left.+8 \alpha_{2} \alpha \beta-\alpha_{1} \beta^{2}+7 \alpha_{2}^{2} \beta\right) n^{2}+\left(-\alpha_{2}^{2} \alpha_{1} \beta-\alpha_{1} \alpha_{2} \beta-\alpha_{1} \beta^{2} \alpha_{2}\right. \\
& +\alpha_{2}^{3} \beta-\alpha_{1} \alpha^{2} \beta-\alpha_{1} \alpha \beta+2 \alpha_{2}^{2} \alpha \beta+\alpha_{2} \alpha \beta-2 \alpha_{1} \alpha_{2} \alpha \beta \\
& \left.\left.+\alpha_{2} \alpha^{2} \beta+\alpha_{2}^{2} \beta+\beta^{2} \alpha_{2}^{2}\right) n\right) t(n+1, n+1)+27 n^{5} \\
& +\left(54 \alpha_{2}+27 \alpha-3 \beta-27 \alpha_{1}+27\right) n^{4} \\
& +\left(3 \alpha_{1} \beta-27 \alpha_{1} \alpha+45 \alpha_{2} \alpha-27 \alpha_{1} \alpha_{2}-\beta+45 \alpha_{2}-4 \alpha \beta\right. \\
& \left.+6+36 \alpha_{2}^{2}-27 \alpha_{1}+18 \alpha+9 \alpha^{2}-\alpha_{2} \beta\right) n^{3} \\
& +\left(-6 \alpha_{1}+8 \alpha_{2}+2 \alpha-\alpha^{2} \beta-18 \alpha_{1} \alpha_{2} \alpha+21 \alpha_{2}^{2} \alpha+21 \alpha_{2}^{2}\right. \\
& +3 \alpha_{2}^{2} \beta-18 \alpha_{1} \alpha+\alpha_{1} \beta-9 \alpha_{1} \alpha^{2}+3 \alpha^{2}-\alpha \beta+24 \alpha_{2} \alpha \\
& -2 \alpha_{1} \alpha_{2} \beta-9 \alpha_{2}^{2} \alpha_{1}+\alpha^{3}-4 \alpha_{2} \alpha \beta+10 \alpha_{2}^{3}-18 \alpha_{1} \alpha_{2} \\
& \left.+12 \alpha_{2} \alpha^{2}+4 \alpha_{1} \alpha \beta\right) n^{2}+\left(-3 \alpha_{1} \alpha_{2} \alpha^{2}+\alpha_{2}^{4}+6 \alpha_{2}^{2} \alpha+2 \alpha_{2}^{2}-2 \alpha_{1} \alpha\right. \\
& +2 \alpha_{2} \alpha-3 \alpha_{1} \alpha^{2}+\alpha_{2}^{3} \beta--\alpha_{2}^{3} \alpha_{1}+3 \alpha_{2}^{3} \alpha-6 \alpha_{1} \alpha_{2} \alpha-\alpha_{1} \alpha_{2} \beta \\
& +\alpha_{2}^{2} \beta-2 \alpha_{1} \alpha_{2}-3 \alpha_{2}^{2} \alpha_{1} \alpha+3 \alpha_{2}^{2} \alpha^{2}+3 \alpha_{2} \alpha^{2}+3 \alpha_{2}^{3}-3 \alpha_{2}^{2} \alpha_{1} \\
& \left.-\alpha_{1} \alpha^{3}-\alpha_{2}^{2} \alpha_{1} \beta+\alpha_{2} \alpha^{3}-\alpha_{2} \alpha \beta+\alpha_{1} \alpha \beta+\alpha_{1} \alpha^{2} \beta-\alpha_{2} \alpha^{2} \beta\right) n \text {. }
\end{aligned}
$$




\section{Список литературы}

[1] Е. М. Никишин, В.Н. Сорокин, Рациональные аппроксимации и ортогональность, Наука, М., 1988 MR 0953788, Zbl 0718.41002.

[2] A. I. Aptekarev, A. Branquinho, W. Van Assche, "Multiple orthogonal polynomials for classical weights", Trans. Amer. Math. Soc., 355:10 (2003), 3887-3914 doi 10.1090/S0002-9947-03-03330-0, MR 1990569, Zbl 1033.33002.

[3] В.А. Калягин, "Аппроксимаций Эрмита-Паде и спектральный анализ несимметричных операторов", Матем. сб., 185:6 (1994), 79-100 Mi sm903, MR 1280397, Zbl 0840.47026.

[4] A. Aptekarev, V. Kaliaguine, "Complex rational approximation and difference operators", Proceedings of the Third International Conference on Functional Analysis and Approximation Theory, vol. I (Acquafredda di Maratea, 1996), Rend. Circ. Mat. Palermo (2) Suppl., 52, 1998, 3-21 MR 1644539, Zbl 0917.47028.

[5] V. Kaliaguine, A. Ronveaux, "On a system of "classical" polynomials of simultaneouse orthogonality", J. Comput. Appl. Math., 67:2 (1996), 207-217 MR 1390180, Zbl 0857.42012.

[6] Дж. Бейкер (мл.), П. Грейвс-Моррис, Аппроксимачии Паде, Ч. 1: Основы теории. Ч. 2: Обобщения и приложения, Мир, М., 1986 MR 0868466, Zbl 0669.30023. 